\title{
From fast to slow light in a resonantly driven absorbing medium
}

\author{
Bruno Macke and Bernard Ségard* \\ Laboratoire de Physique des Lasers, Atomes et Molécules , \\ CNRS et Université Lille 1, 59655 Villeneuve d'Ascq, France
}

(Dated: May 3, 2018)

\begin{abstract}
We theoretically study the propagation through a resonant absorbing medium of a time-dependent perturbation modulating the amplitude of a continuous wave (cw). Modeling the medium as a twolevel system and linearizing the Maxwell-Bloch equations for the perturbation, we establish an exact analytical expression of the transfer function relating the Fourier transforms of the incident and transmitted perturbations. It directly gives the gain and the phase shift undergone in the medium by a harmonic modulation. For the case of a pulse modulation, it enables us to determine the transmission time of the pulse center-of-mass (group delay), evidencing the relative contributions of the coherent and incoherent (population) relaxation. We show that the group delay has a negative value (fast light) fixed by the coherent effects when the cw intensity is small compared to the saturation intensity and becomes positive (slow light) when this intensity increases, before attaining a maximum that cannot exceed the population relaxation time. The analytical results are completed by numerical determinations of the shape of the transmitted pulses in the different regimes.
\end{abstract}

PACS numbers: 42.25.Bs, $42.50 . \mathrm{Hz}, 42.50 \mathrm{~Gy}$

\section{INTRODUCTION}

Convincing demonstrations of fast light in a linear homogeneous medium were performed in the 1980 s by exploiting the steep anomalous dispersion associated with a well-isolated, narrow and strong absorption line of the medium, leading to negative values of the group velocity [1, 2]. Ideally bell-shaped pulses can then propagate moderately distorted in such a way that the maximum of the transmitted pulse occurs before that of the incident pulse. Time-advances exceeding 0.5 times the halfduration at half-maximum of the pulse-envelope have been so evidenced in experiments involving a true shape detection of the latter [2]. See also [3, 4]. More generally, due to the causality principle (originating the KramersKronig relations), fast light is expected every time that the carrier frequency of the pulses coincides with the bottom of a well-marked dip in the medium transmission and the pulse distortion resulting from the first order variations of the transmission and of the group velocity versus frequency cancels when this coincidence is exact [5]. The dip in the transmission may be natural or created in various arrangements involving electromagnetically induced absorption [6, 7], stimulated Raman [8] or Brillouin [9] scattering, etc. The literature on fast light is abundant (for reviews, see, e.g., [10 13]) but it seems that the fractional pulse-advance with moderate distortion reported in [2] has not been overtaken. This is easily explained by remarking that, since the transmission dip at the carrier frequency must be well pronounced, there are spectral regions where the overall transmission of the system is much larger. For obvious reasons of noise (no matter its origin), instability and hypersensitiv-

*Electronic address: bernard.segard@univ-lille1.fr ity to small defects in the incident pulse-shape, the usable transmission-dynamics cannot be too large and this limits the observable fast-light effects in any linear system [14]. The dynamics actually involved in the fast-light experiments does not exceed $45 \mathrm{~dB}$ and is often much lower. The situation is quite different in the slow-light experiments where the medium transmission is maximal at the carrier frequency and can be very low on both sides of this frequency, with transmission-dynamics exceeding $1000 \mathrm{~dB}$ [15, 16].

In the present article, we study the propagation in an absorbing medium of a continuous wave (cw) whose amplitude is pulse-modulated with a low modulation index. The medium is modeled as a homogeneously-broadened two-level system (TLS) [17] with a resonance frequency $\omega_{0}$ and a relaxation time $T_{1}\left(T_{2}\right)$ for the population difference (the coherence). We assume that the $\mathrm{cw}$ is on exact resonance and we are interested in the propagation of the pulsed part of the wave (the pulse) whose optical spectrum is centered on $\omega_{0}$ and maximum at this frequency. The pulse may thus be seen as a continuous superposition of fields symmetrically detuned from the cw field (sidebands) that probe the changes of the TLS properties induced by the $\mathrm{cw}$. The propagation in a strongly driven TLS of two symmetrically detuned fields is a basic problem in nonlinear optics [18, 19]. When the sidebands are associated with an amplitude modulation, it has been experimentally evidenced [20] and theoretically demonstrated [18, 21] that the transmission of the modulation presents a maximum on resonance for strong enough cw intensities. According to our general analysis, the pulse propagation may be slow in such conditions. On the other hand, when the cw intensity is small compared to the saturation intensity, the TLS behaves linearly both for the cw and the pulse, the propagation of which is not modified by the presence of the cw (fast light regime). Our main purpose is to analyze how takes 
place the transition from a fast to a slow light regime when the cw intensity increases. Our calculations take full account of the depletion of the cw intensity during the propagation and are made for arbitrary values of the relaxation times $T_{1}$ and $T_{2}$. The corresponding results (mainly analytical) are new and it seems that the possibility of switching from fast to slow light in a TLS by simply adding a resonant $\mathrm{cw}$ to the pulses has not been previously considered.

The arrangement of our paper is as follows. In Sec. [I] we specify the system under consideration and we establish an exact analytical expression of its transfer function for the time-dependent part of the field envelope. This expression directly gives the gain and the phase shift undergone in the medium by a harmonic modulation, examined in Sec III. The propagation of pulses is studied in Sec. IV] with a special attention paid to the transmission delay of their center-of-mass (identified to the group delay). The analytical results are completed by numerical determination of the envelope of the transmitted pulses. We finally conclude in Sec. $\mathrm{V}$ by summarizing the main results.

\section{TRANSFER FUNCTION OF THE MEDIUM}

As above mentioned we consider the case where the $\mathrm{cw}$ is exactly resonant. The amplitude modulation is then an eigenmode of modulation in the sense that that it is conserved during the propagation [22 24]. We denote $\ell$ the thickness of the medium, $\alpha$ its unsaturated absorption coefficient on resonance for the intensity and $z(0<z<\ell)$ the direction of propagation of the wave assumed to be plane and polarized along the $x$-axis. We write the $x$-component of the electric field as :

$$
E_{x}(z, t)=\operatorname{Re}\left[\mathrm{e}^{i \omega_{0} t} \widetilde{E}(z, t)\right]
$$

where $\widetilde{E}(z, t)$ is the slowly varying field-envelope. As in all the following, $t$ is a local time, that is the real time minus $n_{0} z / c, c$ being the velocity of light in vacuum and $n_{0}$ the refractive-index around $\omega_{0}$ of the eventual host medium. Denoting $\mu$ the dipole matrix element for the transition (chosen real), $R(z, t)=\frac{\mu \widetilde{E}(z, t)}{\hbar}$ the Rabi frequency, $N(z, t)$ the population difference per volume unit $\left(N_{0}\right.$ its value at equilibrium) and $\widetilde{P}(z, t)$ the envelope of the electric polarization induced in the medium, it is convenient to introduce the dimensionless quantities $D=\frac{N}{N_{0}}, P=i \frac{\widetilde{P}}{N_{0} \mu} \sqrt{\frac{T_{1}}{T_{2}}}$ and $E=\frac{\mu \widetilde{E}}{\hbar} \sqrt{T_{1} T_{2}}=R \sqrt{T_{1} T_{2}}$. Note that all these quantities are real for the resonant case considered here and that $I=E^{2}$ is the intensity normalized to the saturation intensity. In such conditions, the Maxwell-Bloch (MB) equations governing the system evolution take the simple form :

$$
\frac{\partial E}{\partial z}=-\frac{\alpha}{2} P
$$

$$
\begin{gathered}
T_{2} \frac{\partial P}{\partial t}=D E-P \\
T_{1} \frac{\partial D}{\partial t}=-P E+(1-D)
\end{gathered}
$$

These equations are easily solved when only the $\mathrm{cw}$ is present. In the following we denote the corresponding values of $E, P, D$ and $I$ by the index $c w$. Combining Eqs. (3) and (4), we find $D_{c w}(z)=1 /\left[1+I_{c w}(z)\right]$ and $P_{c w}(z)=E_{c w}(z) /\left[1+I_{c w}(z)\right]$. Replacing this result in Eq. (2), we get :

$$
\begin{gathered}
\frac{\partial}{\partial z}\left[I_{c w}(z)+\ln I_{c w}(z)+\alpha z\right]=0 \\
I_{\text {out }}+\ln I_{\text {out }}=I_{\text {in }}+\ln I_{\text {in }}-\alpha \ell
\end{gathered}
$$

where $I_{\text {out }}$ and $I_{\text {in }}$ are short hand notations of $I_{c w}(\ell)$ and $I_{c w}(0)$. When a small time-dependent perturbation $\delta E_{i n}(t)$ (also real for an amplitude modulation) is added to the constant amplitude $E_{i n}=\sqrt{I_{i n}}$ of the field of the incident $\mathrm{cw}$, we search for solutions of the MB equations under the form $E(z, t)=E_{c w}(z)+\delta E(z, t), D(z, t)=$ $D_{c w}(z)+\delta D(z, t)$ and $P(z, t)=P_{c w}(z)+\delta P(z, t)$. Taking into account the $\mathrm{cw}$ solution, we get at the first order of perturbation

$$
\begin{gathered}
\frac{\partial(\delta E)}{\partial z}=-\frac{\alpha}{2} \delta P \\
T_{2} \frac{\partial(\delta P)}{\partial t}=E_{c w} \delta D+D_{c w} \delta E-\delta P \\
T_{1} \frac{\partial(\delta D)}{\partial t}=-E_{c w} \delta P-P_{c w} \delta E-\delta D
\end{gathered}
$$

These linear equations can be solved by introducing the Fourier transforms $\Delta E(z, \Omega), \Delta P(z, \Omega)$ and $\Delta D(z, \Omega)$ of $\delta E(z, t), \delta P(z, t)$ and $\delta D(z, t)$ [25]. From Eqs (8) and (9), we get :

$\Delta P(z, \Omega)=\frac{\left[1+i \Omega T_{1}-I_{c w}(z)\right] \Delta E(z, \Omega)}{\left[1+I_{c w}(z)\right]\left[\left(1+i \Omega T_{1}\right)\left(1+i \Omega T_{2}\right)+I_{c w}(z)\right]}$

Replacing this result in Eq.(7) and integrating on $z$, we finally obtain the transfer function $H(\Omega)$ relating $\Delta E_{\text {out }}(\Omega)$ and $\Delta E_{\text {in }}(\Omega)$, the Fourier transforms of $\delta E_{\text {out }}(t)=\delta E(\ell, t)$ and $\delta E_{\text {in }}(t)$. It reads as :

$$
H(\Omega)=\exp \left(\int_{0}^{\ell} f(z, \Omega) d z\right)
$$

where

$$
f(z, \Omega)=\frac{\alpha}{2} \frac{I_{c w}(z)-\left(1+i \Omega T_{1}\right)}{\left[1+I_{c w}(z)\right]\left[\left(1+i \Omega T_{1}\right)\left(1+i \Omega T_{2}\right)+I_{c w}(z)\right]}
$$


is the complex gain factor at the abscissa $z$. By means of Eq.(5), the integration on $z$ in Eq.(11) can be transformed in an integration on $I_{c w}$. We get :

$H(\Omega)=\exp \left[\frac{1}{2} \int_{I_{\text {in }}}^{I_{\text {out }}} \frac{1+i \Omega T_{1}-I_{c w}}{I_{c w}\left[\left(1+i \Omega T_{1}\right)\left(1+i \Omega T_{2}\right)+I_{c w}\right]} d I_{c w}\right]$

and finally

$$
\begin{array}{r}
H(\Omega)=\exp \left[\frac{1+i \frac{\Omega T_{2}}{2}}{1+i \Omega T_{2}} \ln \left(\frac{I_{\text {in }}+\left(1+i \Omega T_{1}\right)\left(1+i \Omega T_{2}\right)}{I_{\text {out }}+\left(1+i \Omega T_{1}\right)\left(1+i \Omega T_{2}\right)}\right)\right. \\
\left.-\frac{\ln \sqrt{I_{\text {in }} / I_{\text {out }}}}{\left(1+i \Omega T_{2}\right)}\right]
\end{array}
$$

where $I_{\text {out }}$ and $I_{\text {in }}$ are related by Eq.(6). This expression of $H(\Omega)$ is the central result of our paper. In the limit $I_{\text {in }} \ll 1, I_{\text {out }} \approx I_{\text {in }} \exp (-\alpha \ell)$ and $H(\Omega)$ is reduced to $\exp \left(-\frac{\alpha \ell}{2\left(1+i \Omega T_{2}\right)}\right)$ which, as expected, is the transfer function of a linear medium with a Lorentzian absorption line [5]. Quite generally, we see that the poles of $H(\Omega)$ are in the half-plane $\operatorname{Im}(\Omega)>0$ and that $H(-\Omega)=H^{\star}(\Omega)$. This ensures that the impulse response $h(t)$, inverse Fourier transform of $H(\Omega)$, cancels for $t<0$ and is real, confirming that the system is causal 25] and that the amplitude modulation is an eigenmode of modulation. At the first order of perturbation considered here, we finally remark that

$I(z, t)=\left[E_{c w}(z)+\delta E(z, t)\right]^{2} \approx I_{c w}(z)+2 \delta E(z, t) \sqrt{I_{c w}(z)}$

This means in particular that a quadratic detection, as currently used in optics, will deliver a time dependent signal $\delta I_{\text {out }}(t)$ proportional to $\delta E_{\text {out }}(t)$ and that the instantaneous modulation indices for the intensity and for the field are such that $\frac{\delta I(z, t)}{I_{c w}(z)}=2 \frac{\delta E(z, t)}{E_{c w}(z)}$ in every point.

\section{TRANSMISSION OF A HARMONIC MODULATION}
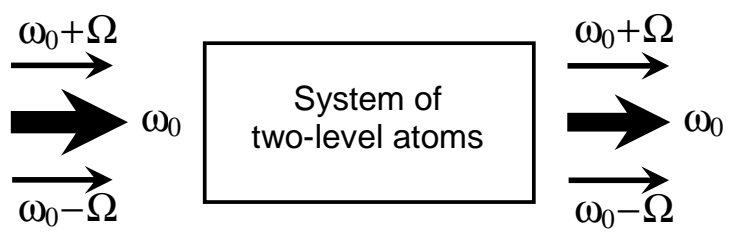

Figure 1: The driving wave (cw) at $\omega_{0}$ can modify the propagation of the pair of sidebands at $\omega_{0} \pm \Omega$.

When the amplitude-modulation is harmonic of frequency $\Omega(\Omega>0)$, the optical field has two sidebands at $\omega_{0} \pm \Omega$ that both act as probes of the system driven at $\omega_{0}$ (Fig,1). The modulus $G(\Omega)$ and the argument $\Phi(\Omega)$ of $H(\Omega)$ are then respectively the gain and the phase-shift of the output amplitude-modulation with respect to the input one. The gain coefficient $g(z, \Omega)=\operatorname{Re}[f(z, \Omega)]$ and the phase-shift coefficient $\varphi(z, \Omega)=\operatorname{Im}[f(z, \Omega)]$ are the corresponding quantities per unit length. Due to the depletion of the cw intensity $I_{c w}$ during the propagation, these latter quantities depend on $z$. Note that the expression of $g(z, \Omega)$ derived from Eq. (12) is consistent with the results given in [18, 21], also as in [20] for the particular case $T_{1}=T_{2}$. Figures 2 and 3 show a set of profiles $g(\Omega)$ and $\varphi(\Omega)$ obtained for $T_{2}=2 T_{1}$ (purely radiative relaxation) with various cw intensities. For $I_{c w} \ll 1$ (fully linear case), we obviously retrieve the gain and dispersion profiles $g(\Omega)=-\frac{\alpha}{2\left(1+\Omega^{2} T_{2}^{2}\right)}$ and $\varphi(\Omega)=\frac{\alpha \Omega T_{2}}{2\left(1+\Omega^{2} T_{2}^{2}\right)}$, associated with a Lorentzian absorption line.

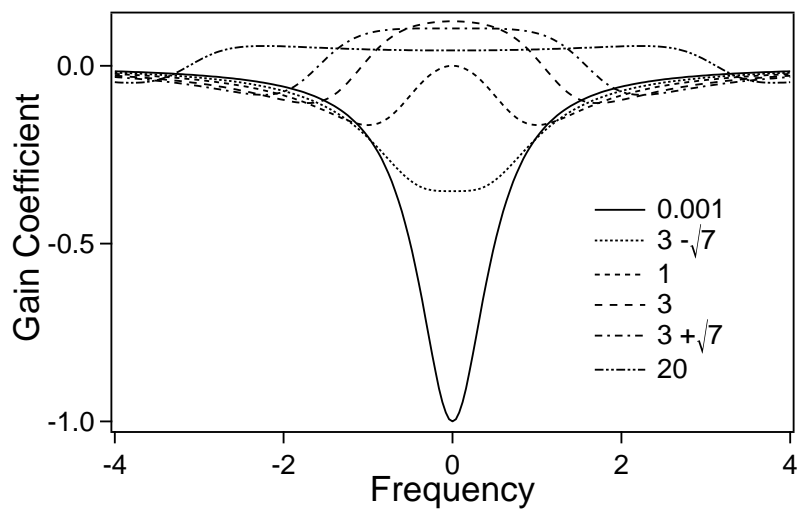

Figure 2: Gain coefficient $g(\Omega)$ in $\alpha / 2$ units as a function of $\Omega$ in $1 / T_{1}$ units for $T_{2}=2 T_{1}$. The different curves are labeled by the corresponding value of the normalized intensity $I_{c w}$.

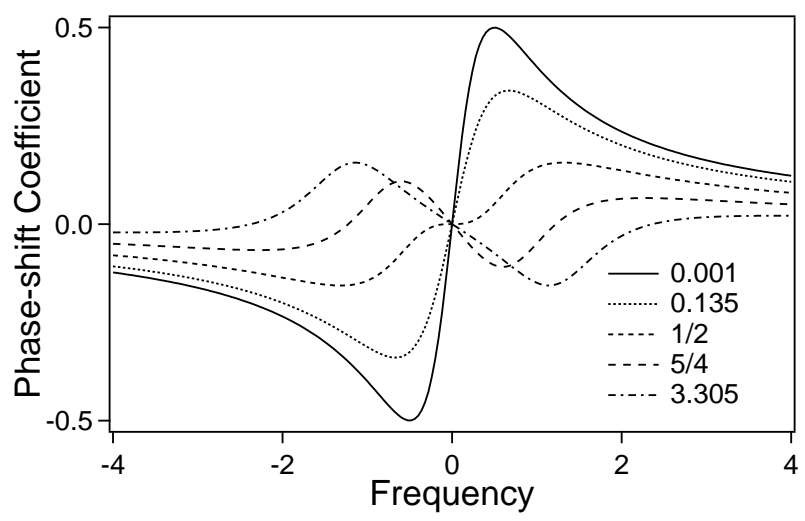

Figure 3: Same as Fig 2 for the phase-shift coefficient $\varphi(\Omega)$ in $\alpha / 2$ units.

The gain coefficient (Fig 2) keeps negative as long as $I_{c w}<1$. It cancels at $\Omega=0$ when $I_{c w}=1$ (absolute $\mathrm{cw}$ intensity equal to the saturation intensity). Beyond, it becomes positive in the frequency range $\Omega<\Omega_{g}=$ $\sqrt{\left(I_{c w}^{2}-1\right) /\left(I_{c w} T_{1} T_{2}+T_{1}^{2}\right)}$, attaining an absolute maximum $\alpha / 16$ for $I_{c w}=3$. When $I_{c w} \gg 1, \Omega_{g} \approx R_{c w}$ , the Rabi frequency associated with the cw field. We also note that, $g(\Omega)$ being an even function, $g(0)=$ 
$\frac{\alpha\left(I_{c w}-1\right)}{2\left(I_{c w}+1\right)^{2}}$ is always an extremum of the gain-coefficient. This extremum is flat for $I_{c w}=b \pm \sqrt{b^{2}-T_{2} / T_{1}}$ with $b=3 / 2+T_{1} / T_{2}+T_{2} / 2 T_{1}$, that is $I_{c w}=3 \pm \sqrt{7}$ when $T_{2}=2 T_{1}$. Despite some common points, the profiles $g(\Omega)$ significantly differ from those obtained by using a probe independent of the driving field [18, 26 29]. They are a bit simpler, probably because the amplitude modulation is an eigenmode of modulation (contrary to the single sideband modulation). On the other hand, the refractive index experienced by the optical probe field, well defined for the single sideband case [30], is not defined for the case of an amplitude modulation where the probe field has two frequency-components at $\omega_{0} \pm \Omega$ that are not independent.

The phase-shift coefficient $\varphi(\Omega)$ (Fig. 3) has a maximum $\varphi_{\max }=\alpha / 4$ (phase advance) at $\Omega=1 / T_{2}$ for $I_{c w} \ll 1$ and an absolute minimum $\varphi_{\text {min }} \approx-0.078 \alpha$ (phase lag) at $\Omega \approx 1.14 / T_{1}$ for $I_{c w} \approx 3.3$. On the other hand, the slope $\left.\frac{d \varphi}{d \Omega}\right|_{\Omega=0}$ at the origin is also maximal for $I_{c w} \ll 1$. This slope is half-maximum, cancels and attains its (negative) minimum, for $I_{c w}$ respectively equal to $0.135,1 / 2$ and $5 / 4$.

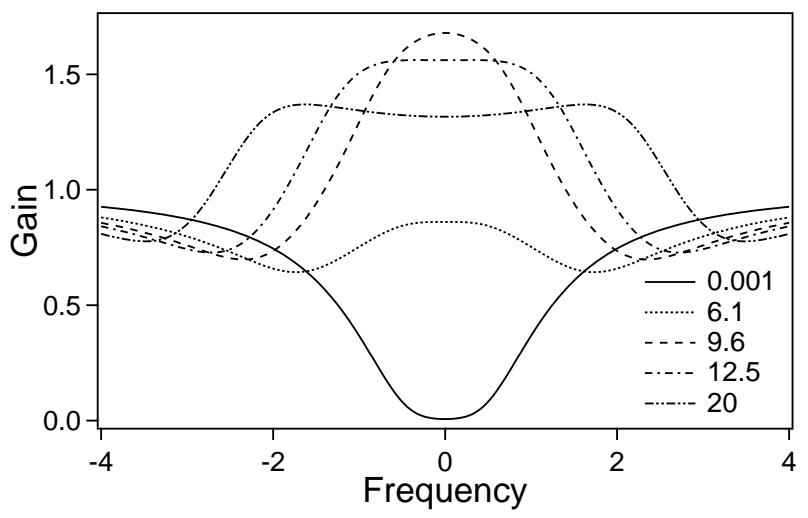

Figure 4: Gain $G(\Omega)$ as a function of $\Omega$ in $1 / T_{1}$ units for $T_{2}=2 T_{1}$ and $\alpha \ell=10$. The different curves are labeled by the corresponding value of the normalized intensity $I_{i n}$.

Representative profiles of the (overall) gain $G(\Omega)$ and phase-shift $\Phi(\Omega)$ are shown Fig 4 and Fig 5 for $\alpha \ell=10$, with various intensities $I_{\text {in }}$ chosen according to criteria analogue to those used Fig, 2 and Fig 3. The optical thickness retained is of the order of that actually used in [2]. When $I_{\text {in }} \ll 1, G(\Omega)=\exp \left[-\frac{\alpha \ell}{2\left(1+\Omega^{2} T_{2}^{2}\right)}\right]$ and $\Phi(\Omega)=\frac{\alpha \ell \Omega T_{2}}{2\left(1+\Omega^{2} T_{2}^{2}\right)}$. Except for this fully linear case, Eqs. (11) and (12) show that $G(\Omega)$ and $\Phi(\Omega)$ are strongly affected by the depletion of the cw intensity in the medium, often not taken into account in the literature. This point is illustrated Fig 6 where we give the profile $G(\Omega)=\exp [\ell g(\Omega)]$ which would be obtained by neglecting the cw depletion, i.e. by taking $I_{c w}(z)=I_{\text {in }}$ everywhere. The depletion obviously forces one to use larger incident $\mathrm{cw}$ intensities to obtain similar gain pro-

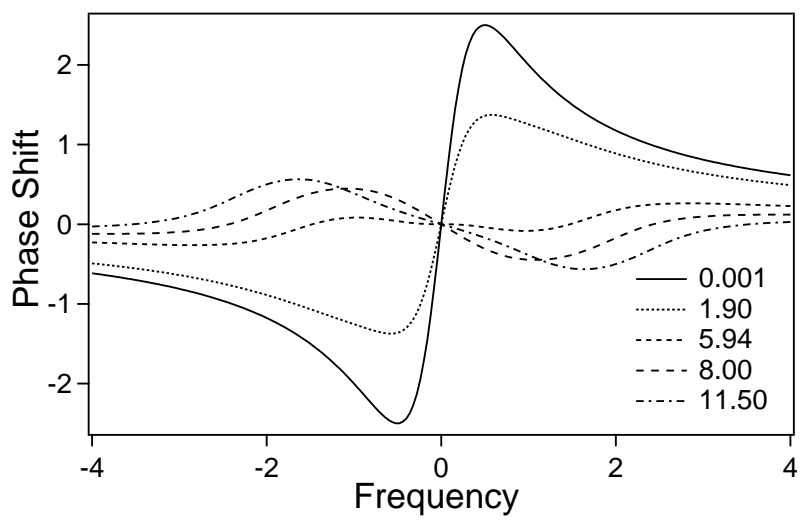

Figure 5: Same as Fig 4 for the phase-shift $\Phi(\Omega)$. The maximum and minimum phase-shift are respectively $\alpha \ell / 4=2.5 \mathrm{Rd}$ at $\Omega=1 / T_{2}$ for $I_{i n} \ll 1$ and $-0.56 \mathrm{Rd}$ at $\Omega=1.68 / T_{1}$ for $I_{\text {in }} \approx 11.5$.

files and the maximum gain is smaller. The difference is more important when the optical thickness $\alpha \ell$ is very large. From Eqs. (6) and (14), we find that the maximum gain then tends to $\sqrt{\alpha \ell} / 2$ whereas it equals $\exp [\alpha \ell / 16]$ when the depletion is neglected. There are obviously similar effects on the phase-shift $\Phi(\Omega)$.

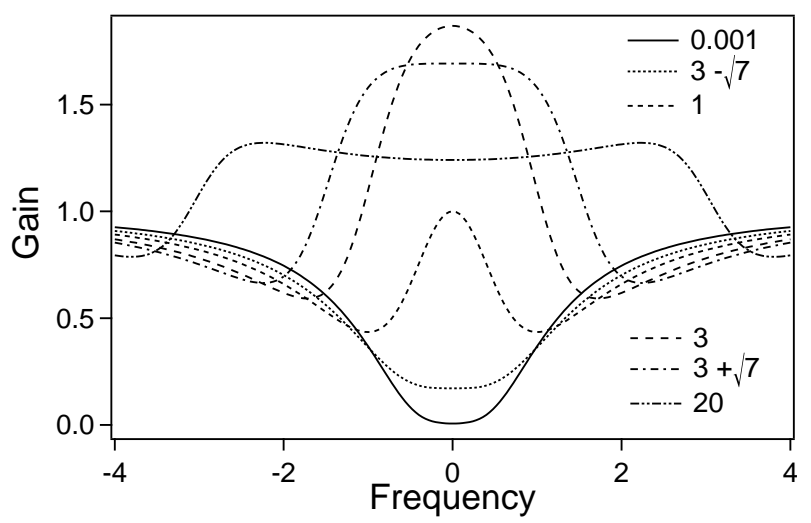

Figure 6: Same as Fig 4 when the depletion of the driving wave is not taken into account.

The previous results take a very simple form in the limit case where $T_{2}$ is negligible compared to $T_{1}$. The MB equations are then reduced to the rate equations [17] that provide a good description of the propagation of light in numbers of saturable absorbers 3136$]$. Putting $T_{2} \approx 0$ in Eqs. (12) and (14), we get

$$
\begin{gathered}
f(z, \Omega)=-\frac{\alpha}{2\left(1+I_{c w}\right)}+\frac{\alpha I_{c w}}{\left(1+I_{c w}\right)^{2}}\left(\frac{1}{1+i \frac{\Omega T_{1}}{1+I_{c w}}}\right) \\
H(\Omega)=\sqrt{\frac{I_{\text {out }}}{I_{\text {in }}}}\left(\frac{1+I_{\text {in }}+i \Omega T_{1}}{1+I_{\text {out }}+i \Omega T_{1}}\right)
\end{gathered}
$$

Apart from the term $-\frac{\alpha}{2\left(1+I_{c w}\right)}$ independent of $\Omega$, the complex gain factor $f(z, \Omega)$ is identical to that 
of a Lorentzian gain line of half-width at half maximum $\left(1+I_{c w}\right) / T_{1}$ with a gain-coefficient on resonance $\alpha I_{c w} /\left(1+I_{c w}\right)^{2}$ for the amplitude. During the propagation in the medium, the modulation index is magnified whereas the phase of the modulation is delayed [35-38]. The magnification $K(\Omega)$ of the modulation index and the phase lag $L(\Omega)=-\Phi(\Omega)$ for both the amplitude and the intensity modulations are easily deduced from Eq.(17). We get :

$$
\begin{aligned}
& K(\Omega)=G(\Omega) \sqrt{\frac{I_{\text {in }}}{I_{\text {out }}}}=\sqrt{\frac{\left(1+I_{\text {in }}\right)^{2}+\Omega^{2} T_{1}^{2}}{\left(1+I_{\text {out }}\right)^{2}+\Omega^{2} T_{1}^{2}}} \\
& L(\Omega)=\tan ^{-1}\left(\frac{\Omega T_{1}\left(I_{\text {in }}-I_{\text {out }}\right)}{\left(1+I_{\text {in }}\right)\left(1+I_{\text {out }}\right)+\Omega^{2} T_{1}^{2}}\right)
\end{aligned}
$$

The phase lag, always positive for this "incoherent" case, attains its maximum $L_{\max }=$ $\tan ^{-1}\left[\left(I_{\text {in }}-I_{\text {out }}\right) / 2 \sqrt{\left(1+I_{\text {in }}\right)\left(1+I_{\text {out }}\right)}\right] \quad$ for $\Omega T_{1}=\sqrt{\left(1+I_{\text {in }}\right)\left(1+I_{\text {out }}\right)}$. We remark that $L_{\max }<\pi / 2$, the upper limit being approached when $I_{\text {in }} \gg 1$ and $I_{\text {out }} \ll 1$, that is for $\alpha \ell \rightarrow \infty$. Consequently the time delay of the output modulation can never exceed a quarter of the modulation period $T=2 \pi / \Omega$ (about an eighth for $\alpha \ell=10$ ). Since the work reported in [39], numerous slow-light experiments performed in saturable media have been analyzed by invoking hole burning via coherent population oscillations (CPO), resulting in a reduction of the group velocity. In fact, a more direct analysis is provided by the basic model of saturable absorber 33] and, as shown in detail in [36], the signals observed in most CPO experiments can be perfectly reproduced by means of Eqs. (18) and (19), eventually extended to take into account inhomogeneous effects.

\section{PULSE PROPAGATION}

Strictly speaking a harmonic modulation does not contain any information and, e.g., a time-delay of $T / 8$ as considered at the end of the previous section can also be seen as a time-advance of $7 T / 8$. Unambiguous demonstrations of fast or slow light require to use pulses of finite duration and energy. An important parameter to characterize the propagation is then the transmission-time of the centerof-mass of the pulse envelope. Following the use in signal theory 25], we define the center-of-mass of a signal $y(t)$ of normalized area as $\int_{-\infty}^{\infty} t y(t) d t$. A direct application of the moment theorem [25] shows then that the transmission-time of the center-of-mass of the pulse envelope is equal to the group delay $\tau_{g}=-\left.\frac{d \Phi}{d \Omega}\right|_{\Omega=0}$, whatever the pulse distortion may be [5]. On the other hand $-\left.\frac{d \varphi}{d \Omega}\right|_{\Omega=0}$ appears as the transmission delay or group delay per unit length, equal in our local time picture to $1 / v_{g}-n_{0} / c \approx 1 / v_{g}$, where $v_{g}$ is the group velocity. We emphasize that, due to the cw depletion, $v_{g}$ is not uniform. From Eq.(14), we get $\tau_{g}=\tau_{1}+\tau_{2}$ with

$$
\begin{gathered}
\tau_{1}=T_{1}\left(\frac{1}{I_{\text {out }}+1}-\frac{1}{I_{\text {in }}+1}\right) \\
\tau_{2}=T_{2}\left(\frac{1}{I_{\text {out }}+1}-\frac{1}{I_{\text {in }}+1}-\ln \sqrt{\frac{1+1 / I_{\text {out }}}{1+1 / I_{\text {in }}}}\right)
\end{gathered}
$$

$\tau_{1}\left(\tau_{2}\right)$, proportional to $T_{1}\left(T_{2}\right)$, may be considered as the contribution to the group delay of the incoherent (coherent) effects. We see that the incoherent or population effects always lead to a delay whereas the coherent ones mainly lead to a much larger time-advance (Fig [7), at least when $T_{2}$ is comparable to $T_{1}$ (the coherent effects obviously disappear in a saturable absorber where $\left.T_{2} \approx 0\right)$.

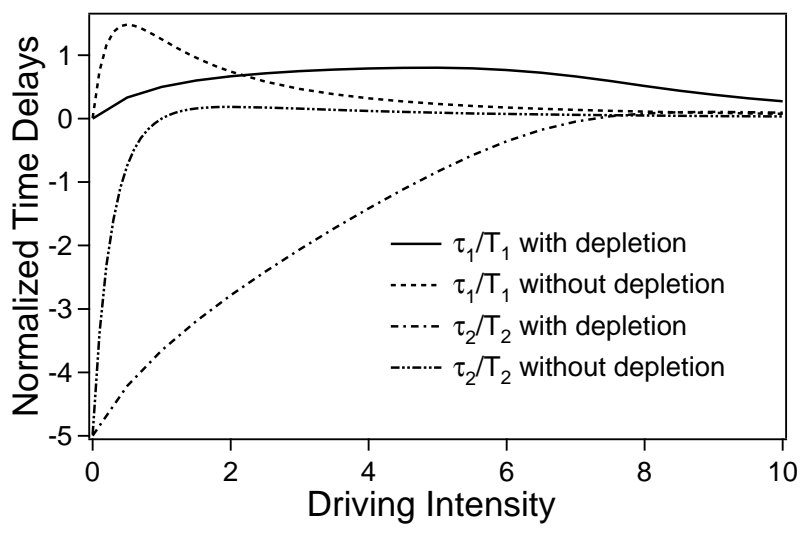

Figure 7: Normalized coherent and incoherent contributions to the group delay as a function of the incident driving intensity $I_{i n}$, depending whether the cw depletion $(\alpha \ell=10)$ is taken into account or not.

Before commenting further on Eqs. (20) and (21), consider the simpler results obtained when the depletion of the driving wave is neglected $\left(I_{c w}=I_{i n}\right.$ everywhere). From Eq.(12), we then get :

$$
\begin{gathered}
\tau_{1}=T_{1} \frac{\alpha \ell I_{i n}}{\left(1+I_{i n}\right)^{3}} \\
\tau_{2}=-T_{2} \frac{\alpha \ell\left(1-I_{i n}\right)}{2\left(1+I_{i n}\right)^{3}}
\end{gathered}
$$

$\tau_{1} / T_{1}$, negligible for $I_{\text {in }} \ll 1$, is maximum for $I_{\text {in }}=1 / 2$ whereas $\tau_{2} / T_{2}$ starts from the large negative value $-\alpha \ell / 2$ before attaining a small positive maximum for $I_{i n}=2$. The total group delay cancels for $I_{\text {in }}=T_{2} /\left(T_{2}+2 T_{1}\right)$ and is maximum for $I_{i n}=\left(2 T_{2}+T_{1}\right) /\left(T_{2}+2 T_{1}\right)$ that is respectively for $I_{i n}=1 / 2$ and $I_{i n}=5 / 4$ when the relaxation is purely radiative (see Fig[3). As expected, taking into account the depletion of the $\mathrm{cw}$ intensity does not modify the delays for $I_{i n} \ll 1$ but shifts the curves $\tau_{1}\left(I_{i n}\right)$ 
and $\tau_{2}\left(I_{i n}\right)$ to larger $I_{\text {in }}$, dramatically spreads them and reduces the amplitude of their maximum (Fig[7). Equation (20) shows that $\tau_{1}$ cannot overtake $T_{1}$, this value being approached when $I_{\text {in }} \gg 1$ and $I_{\text {out }} \ll 1$, that is for $\alpha \ell$ extremely large [35]. An estimate of the maximum of $\tau_{1}$ for large but realistic values of $\alpha \ell$ can be obtain by anticipating that it is attained for $I_{\text {out }} \ll 1$. From Eq.(6) we get then $I_{\text {out }} \approx I_{\text {in }} \exp \left(I_{\text {in }}-\alpha \ell\right)$ and

$$
\tau_{1} \approx T_{1}\left(\frac{1}{I_{\text {in }} \exp \left(I_{\text {in }}-\alpha \ell\right)+1}-\frac{1}{I_{\text {in }}+1}\right)
$$

The maximum is obtained for $\left(I_{i n}+1\right)^{3} \approx$ $\exp \left(\alpha \ell-I_{\text {in }}\right)$. For $\alpha \ell=10\left(\right.$ Fig [7), we find $I_{\text {in }} \approx 4.8$ and, putting this value in Eq.(24), $\max \left(\tau_{1} / T_{1}\right) \approx 0.80$ in perfect agreement with the exact value. By the same method we find $\max \left(\tau_{1} / T_{1}\right) \approx 0.995$ for $\alpha \ell=200$. An upper bound to the total group delay can be obtained by using the method of the Lagrange multipliers, perfectly adapted to the search of a maximum under constraint, here fixed by Eq.(6). Following the procedure exactly as exposed in the original work of Lagrange [40], we find an extra relation between $I_{\text {in }}$ and $I_{\text {out }}$ at the maximum of $\tau_{g}$, namely

$$
\frac{I_{\text {in }}}{I_{\text {out }}}=\left(\frac{I_{\text {in }}+1}{I_{\text {out }}+1}\right)^{3}\left(\frac{2 T_{1}+T_{2}\left(1-1 / I_{\text {out }}\right)}{2 T_{1}+T_{2}\left(1-1 / I_{\text {in }}\right)}\right)
$$

A numerical exploration shows that the upper bound to the group delay is attained when $I_{i n}$ is extremely large whereas $I_{\text {out }}$ keeps finite. Equation (25) takes then the form

$$
\left(1+I_{\text {out }}\right)^{3} \approx I_{\text {in }}^{2}\left[I_{\text {out }}-T_{2} /\left(2 T_{1}+T_{2}\right)\right]
$$

which can be verified if and only if $I_{\text {out }} \approx T_{2} /\left(2 T_{1}+T_{2}\right)$. Injecting this result in the general expression of $\tau_{g}$, we finally find :

$$
\sup \left[\max \left(\tau_{g}\right)\right]=T_{1}+\frac{T_{2}}{2}-T_{2} \ln \sqrt{2\left(1+\frac{T_{1}}{T_{2}}\right)}
$$

When $T_{2} \approx 0$ (saturable absorber), we obviously retrieve the upper bound $T_{1}$ for $\tau_{g}$ whereas this upper bound is $(2-\ln 3) T_{1} \approx 0.90 T_{1}$ when the relaxation is purely radiative $\left(T_{2}=2 T_{1}\right)$. For the latter case $\max \left(\tau_{g} / T_{1}\right)$ is only 0.68 for $\alpha \ell=10$, significantly below its upper limit, and raises to 0.89 for $\alpha \ell=200$.

As before mentioned, the group delay $\tau_{g}$ is the transmission-delay of the pulse center-of-mass, as large as the pulse distortion may be. On the other hand, the envelope $\delta E_{\text {out }}(t)$ of the transmitted pulse is simply the inverse Fourier transform of $H(\Omega) \Delta E_{\text {in }}(\Omega)$. When the duration $\tau_{p}$ of the incident pulse is long enough, $\Delta E_{i n}(\Omega)$ is concentrated around $\Omega=0$ where $H(\Omega) \approx \sqrt{I_{\text {in }} / I_{\text {out }}} \exp \left(-i \Omega \tau_{g}\right)$. We then get $\delta E_{\text {out }}(t)=$ $\sqrt{I_{\text {in }} / I_{\text {out }}} \delta E_{\text {in }}\left(t-\tau_{g}\right)$. The whole envelope is multiplied by $\sqrt{I_{\text {in }} / I_{\text {out }}}$ and time shifted by $\tau_{g}$, without any distortion. Strictly speaking, this solution is only valid for a very long pulse and is not really interesting insofar as the time delay is then negligible compared to the pulse duration. For finite $\tau_{p}$, there is always some pulse-distortion whose importance depends in particular on the transmission dynamics (as defined in the introduction). If, as usual, the incident pulse is bell-shaped and symmetric with a maximum at $t=0$, the envelope of the transmitted pulse will be dissymmetric with a maximum at a time $\tau_{m}$ such that $\tau_{m} / \tau_{g}<1$, its center-of-mass keeping exactly at the time $\tau_{g}$ no matter the pulse duration. The challenge in the slow or fast light experiments is to obtain a fractional delay or advance $\left|\tau_{m}\right| / \tau_{p}$ as large as possible, with moderate distortion. $\tau_{m}$ and $\tau_{g}$ are then comparable. The following figures are obtained by using standard techniques of Fast Fourier Transform. The incident pulse is Gaussian and $\tau_{p}$ is its half-duration at half-maximum.

Figures 8 , 9 and 10 show typical shapes of the transmitted pulse for $T_{2}=2 T_{1}$ (radiative relaxation) and $\alpha \ell=10$.

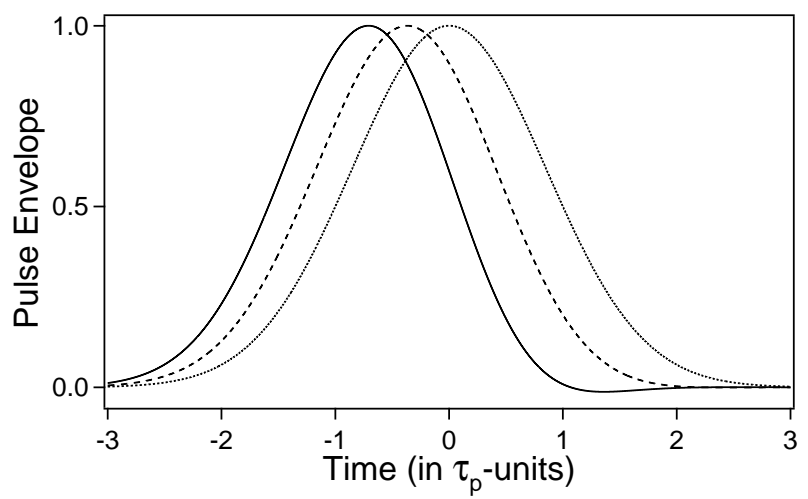

Figure 8: Normalized pulse envelopes obtained for $\alpha \ell=10$ and $T_{2}=2 T_{1}$, when the group advance $-\tau_{g}$ is maximum (solid line) and half-maximum (dashed line). $\tau_{p}$ is chosen such that the fractional advance of the pulse maximum $-\tau_{m} / \tau_{p}$ is significant whereas the distortion remains moderate $\left(\tau_{p} / T_{1}=\right.$ 12.5). For the first case, $\tau_{m} / \tau_{g}=0.88$ and $-\tau_{m} / \tau_{p}=0.71$. For the second case $\tau_{m} / \tau_{g}=0.91$ and $-\tau_{m} / \tau_{p}=0.37$. The envelope of the incident pulse is given for reference (dotted line).

Figures 8 and 9 are obtained for $I_{i n}=0.001$ and $I_{\text {in }}=1.9$ leading respectively to a group advance $-\tau_{g}$ nearly equals to its maximum $\alpha \ell T_{2} / 2$ and the half of this value. The corresponding transmission dynamics is respectively $43 \mathrm{~dB}$ and $26 \mathrm{~dB}$. On Fig $8, \tau_{p}$ has been chosen in order that the fractional advance $-\tau_{m} / \tau_{p}$ is significant (respectively 0.71 and 0.37 ) and the distortion keeps moderate $\left(\tau_{m} / \tau_{g}\right.$ respectively equals 0.88 and 0.91). When the pulse duration is shortened, the fractional advance increases but not as much as one could expect (respectively up to 1.1 and 0.68 ) and this is paid by a dramatic distortion of the transmitted pulse (Fig 9).

Figure 10 is obtained for $I_{i n} \approx 8.0$, leading to the largest normalized group delay $\left(\tau_{g} / T_{1} \approx 0.68\right)$. The cor- 


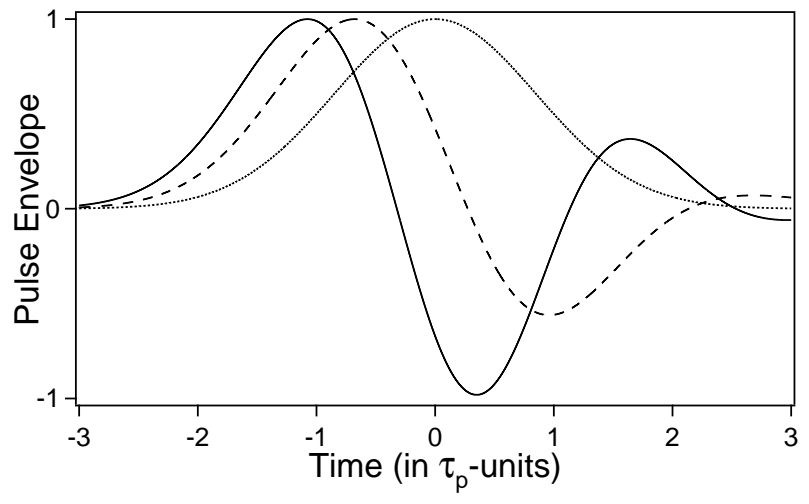

Figure 9: Same as Fig.8 when the pulse durations are those maximizing the fractional advance $-\tau_{m} / \tau_{p}$. For the solid line $\tau_{p} / T_{1}=4.1, \tau_{m} / \tau_{g}=0.44$ and $-\tau_{m} / \tau_{p}=1.1$. For the dashed line $\tau_{p} / T_{1}=2.8, \tau_{m} / \tau_{g}=0.38$ and $-\tau_{m} / \tau_{p}=0.68$.

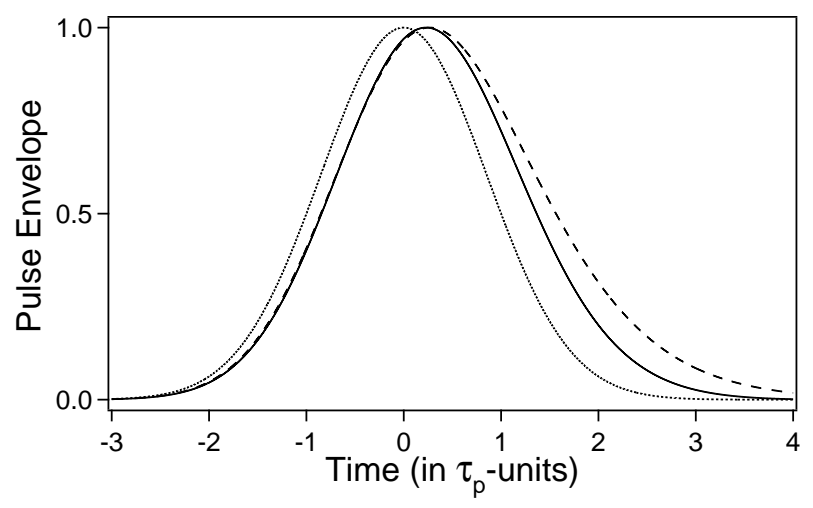

Figure 10: Normalized pulse envelopes obtained for $\alpha \ell=10$ and $T_{2}=2 T_{1}$, when the group delay $\tau_{g}$ is maximum. For the solid line $\tau_{p} / T_{1}=2.27, \tau_{m} / \tau_{g}=0.77$ and $\tau_{m} / \tau_{p}=0.23$. For the dashed line $\tau_{p} / T_{1}=1.44, \tau_{m} / \tau_{g}=0.56$ and $\tau_{m} / \tau_{p}=$ 0.27. The envelope of the incident pulse is given for reference (dotted line).

responding transmission dynamics is small $(7 \mathrm{~dB})$. This explains in part that, even when the pulse duration is optimized (dashed line), the fractional delay $\tau_{m} / \tau_{p}$ does not exceed 0.27 . Another reason is that the coherent relaxation negatively contributes to the group delay (see Fig[7).

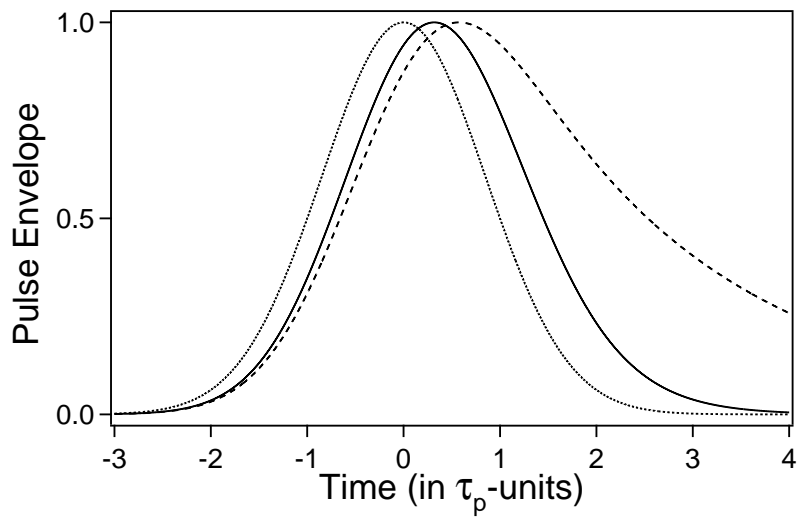

Figure 11: Same as Fig 10 when $T_{2} \approx 0$ (saturable absorber). For the solid line $\tau_{p} / T_{1}=2.0, \tau_{m} / \tau_{g}=0.79$ and $\tau_{m} / \tau_{p}=$ 0.32 . For the dashed line $\tau_{p} / T_{1}=0.44, \tau_{m} / \tau_{g}=0.31$ and $\tau_{m} / \tau_{p}=0.57$.

For a saturable absorber $\left(T_{2} \approx 0\right)$ of same optical thickness $\alpha \ell$, the maximal group delay, now attained for $I_{\text {in }} \approx 4.8$, is upgraded to $0.80 T_{1}$ (see Fig 7) whereas the transmission dynamics attains $15 \mathrm{~dB}$. Figure 11] shows the pulse envelopes obtained for two different pulse durations. As expected, the maximum fractional delay, obtained for $\tau_{p} \approx 0.44 T_{1}$ (dashed line), is significantly larger than for the case of radiative relaxation (more than two times larger). We however remark that the corresponding fall of the pulse is considerably lengthened. An extensive study of the pulse distortion in this particular system can be found in [35].

\section{CONCLUSION}

In their letter appeared in 1982 and soberly entitled "Linear Pulse Propagation in an Absorbing Medium", Chu and Wong [1] paved the way for the now-called fastlight experiments. We have examined in the present article how the propagation is modified when the absorbing medium is driven by a cw. Modeling the absorbing medium as an ensemble of two-level atoms, we have more specifically considered the case where both pulses and $\mathrm{cw}$ are on exact resonance, a condition simply realized by pulse-modulating the cw amplitude, with a low modulation index. This particular arrangement eliminates the pulse-distortion associated with the first order variations of the absorption and of the group delay versus frequency. The basic result of our paper is the exact analytical expression of the transfer function relating the Fourier transforms of the incident and transmitted modulations for arbitrary values of the coherent and incoherent relaxation times $(\mathrm{Eq} 14)$. It shows the importance of the effects resulting from the depletion of the cw intensity along the propagation. When the modulation is only harmonic (as in numerous experiments), it directly gives the gain and the phase shift undergone by the modulation. They significantly depart from those obtained with 
a unique probe field (single sideband modulation). When the modulation is actually pulsed, the transmission delay of the pulse center-of-mass, identified to the group delay, is deduced from the transfer function by a simple calculation of derivative. A remarkable point is that the group delay is the sum of two terms, respectively proportional to the coherent and incoherent relaxation times. These two terms being mainly of opposite sign and depending differently on the cw intensity, this explains why the transmission delay, strongly negative when the cw intensity is low, may become (slightly) positive when the latter increases. Finally the numerical determination of the pulse shapes confirms a general property of the fast and slow light systems, namely that significant advances or delays with moderate distortion can only be obtained in media with a large transmission dynamics. Though our study is only theoretical, it is illustrated for a realistic value of the optical thickness, comparable to that actually used in the microwave experiment reported in [2]. In the optical domain, suitable optical thickness and time-scale could probably be obtained by using an ensemble of cold atoms. We expect that our theoretical work will stimulate such an experiment.
[1] S. Chu and S. Wong, Phys. Rev. Lett. 48, 738 (1982).

[2] B. Ségard and B. Macke, Phys. Lett. 109A, 213 (1985).

[3] H. Tanaka, H. Niwa, K. Hayami, S. Furue, K. Nakayama, T. Kohmoto, M. Kunitomo, and Y. Fukuda, Phys. Rev. A 68, 053801 (2003).

[4] W. G. A. Brown, R. McLean, A. Sidorov, P. Hannaford, and A. Akulshin, J. Opt. Soc. Am. B 25, C82 (2008).

[5] B. Macke and B. Ségard, Eur. Phys. J. D 23,125 (2003).

[6] A. M. Akulshin, S. Barreiro and A. Lezama, Phys. Rev. Lett. 83, 4277 (1999).

[7] A. M. Akulshin, A. Cimmino, and G. I. Opat, Quantum Electronics, 32, 567 (2002).

[8] M. D. Stenner, D. J. Gauthier, and M. A. Neifeld, Nature 425, 695 (2003).

[9] M. González-Herráez, K. Y.Song, and L. Thévenaz, Appl. Phys. Lett. 87, 081113 (2005).

[10] R. Boyd and D. Gauthier, Progress in Optics, 43, 497 (2002).

[11] P. W. Milonni, Fast Light, Slow Light and Left-Handed Light (IOP, Bristol, 2005).

[12] R. W. Boyd and P. Narum, J.Mod.Optics, 54, 2403 (2007).

[13] L. Thévenaz, Nature Photonics 2, 474 (2008).

[14] B. Macke, B. Ségard, and F. Wielonsky, Phys. Rev. E 72, 035601(R) (2005).

[15] A. Kasapi, M. Jain, G. Y. Yin, and S. E. Harris, Phys. Rev. Lett. 74, 2447 (1995).

[16] R. M. Camacho, M. V. Pack, J. C. Howell, A. Schweinsberg and R.W. Boyd, Phys. Rev. Lett. 98, 153601 (2007).

[17] L. Allen and J. H. Eberly, Optical Resonance and Twolevel Atoms (Dover, New York 1987).

[18] M. Sargent III, Physics Reports 43, 223 (1978).

[19] A.V. Sharypov, A. Eilam, A.D. Wilson-Gordon, and H. Friedmann, Phys. Rev. A 81, 013829 (2010).

[20] B. Senitzky, G. Gould, and S. Cutler, Phys. Rev. 130, 1460 (1963).

[21] S. D. Tvogorov, V. G. Fedoseev, and K. N. Yugay, Optika Atmosfery 4, 625 (1991) [Atmospheric and Oceanic Optics 4, 453 (1991)].

[22] L. W. Hillman, R. W. Boyd, and C. R. Stroud, Opt. Lett.
7, 426 (1982).

[23] H. L. Fragnito, E. Palange, C. H. Brito Cruz, and F. DeMartini, Phys. Rev. A 29, 2176 (1984).

[24] M. A. Kramer, R. W. Boyd, L. W. Hillman, and C. R. Stroud, J. Opt. Soc. Am. B 2, 1444 (1985).

[25] Throughout this paper we use the definitions, sign conventions and classical results of the signal theory. See, e.g., A. Papoulis, Signal Analysis (Mc Graw Hill, New York 1988).

[26] S. Haroche and F. Hartmann, Phys. Rev. A 6, 1280 (1972).

[27] A. M. Bonch-Bruevich, V. A. Khodovoi, and N. A. Chigir', Zh. Eksp. Teor. Fiz. 67, 2069 (1974) [Sov. Phys. JETP 40, 1027 (1975)].

[28] F. Y. Wu, S. Ezekiel, M. Ducloy, and B. R. Mollow, Phys. Rev. Lett. 38, 1077 (1977).

[29] R.W. Boyd, M.G. Raymer,P. Narum, and D.J. Harter, Phys. Rev. A 24, 411 (1981).

[30] T. Quang and H. Freedhoff, Phys. Rev. A 48, 3216 (1993)

[31] A. C. Selden, Br. J. Appl. Phys. 18, 743 (1967).

[32] A. C. Selden, J. Phys. D : Appl. Phys. 3, 1935 (1970).

[33] V. S. Zapasskii and G. G. Kozlov, Optics and Spectroscopy 100, 419 (2006).

[34] G. Piredda and R. W. Boyd, J. Eur. Opt. Soc. 2, 07004 (2007).

[35] B. Macke and B. Ségard, Phys. Rev. A 78, 013817 (2008).

[36] A. C. Selden, Optics and Spectroscopy 106, 881 (2009).

[37] A. C. Selden, Electron. Lett. 7, 287 (1971).

[38] L. W. Hillman, R. W. Boyd, J. Krasinski, and C. R. Stroud, Opt. Commun. 45, 416 (1983).

[39] M. S. Bigelow, N. N. Lepeshkin, and R.W. Boyd, Phys. Rev . Lett. 90, 113903 (2003). A review of the subsequent CPO experiments, analyzed in terms of saturable absorption, can be found in the reference [36] of the present paper.

[40] J.L. de la Grange, Méchanique Analitique (Dusart, Paris, 1788), pp 65-66. Available for free consultation at http://gallica.bnf.fr/ark:/12148/bpt6k862625. 